01

\title{
К теории генерации нелинейного фотоакустического сигнала при газомикрофонной регистрации
}

\author{
(C) Т.Х. Салихов, ${ }^{1}$ У. Мадвалиев, ${ }^{2}$ Д.М. Шарифров, ${ }^{3}$ Х.Ш. Туйчиев ${ }^{4}$ \\ ${ }^{1}$ Таджикский национальный университет, \\ 734025 Душанбе, Таджикистан \\ 2 Физико-технический институт им. С.У. Умарова АН Таджикистана, \\ 734063 Душанбе, Таджикистан \\ ${ }^{3}$ Евразийский национальный университет им. Л.Н. Гумилева, \\ 010008 Нур-Султан, Казахстан \\ ${ }^{4}$ Таджикский государственный педагогический университет им. С. Айни, \\ 734025 Душанбе, Таджикистан \\ e-mail: tsalikhov@mail.ru
}

Поступило в Редакцию 20 марта 2021 г.

В окончательной редакции 21 июня 2021 г.

Принято к публикации 22 июня 2021 г.

\begin{abstract}
Предложена теория генерации первых двух гармоник нелинейного фотоакустического сигнала твердотельным образцом с произвольным значением теплопроводности. Для предельных случаев (термически тонких и толстых образцов) получены достаточно простые выражения зависимости амплитуды возбуждаемого фотоакустического сигнала от степени черноты образца и теплофизических параметров образца, газа и подложки, включая их термические коэффициенты.
\end{abstract}

Ключевые слова: фотоакустика, тепловая нелинейность, вторая гармоника.

DOI: $10.21883 / J T F .2021 .11 .51518 .69-21$

\section{Введение}

Различные варианты линейной теории лазерной генерации звуковых волн одно- и двухслойными образцами в фотоакустической (ФА) камере, когда регистрация сигнала проводится микрофонным способом, предложены в [1-4]. Существующие многочисленные теоретические и экспериментальные работы показали, что параметры ФА сигнала насыщены информацией о физических величинах конденсированных сред, включая наносистемы (см., например, [5-11]). Основным механизмом возбуждения акустических волн в этом случае является тепловая, обусловленная периодическим изменением теплового потока, поступающего из образца в газовый слой, модель теплового акустического поршня [1]. Известно, что при выполнении ФА эксперимента, как правило, используется лазерный луч, пространственное распределение которого является гауссовым, и с ростом его интенсивности $I_{0}$ происходит значительное повышение температуры образца, благодаря чему все физические величины среды становятся зависящими от температуры, и эту зависимость принято называть тепловой нелинейностью (ТН) [7].

Особенности возбуждения нелинейных составляющих тепловых волн, включая и вторую гармонику (ВГ), достаточно подробно исследованы в [12-14]. Между тем сравнительно недавно было показано [15], что разрешение ФА микроскопа на ВГ существенно превышает разрешение обычного ФА микроскопа на основной гармонике $(\mathrm{OГ)} \mathrm{и} \mathrm{и} \mathrm{это} \mathrm{позволило} \mathrm{авторам} \mathrm{реализовать}$ визуализацию биологических образцов. По существу, появилась принципиально новая возможность для визуализации сверхвысокого разрешения биологических материалов, что является существенным потенциалом для развития исследований нелинейного ФА отклика образцов.

Для случая, когда регистрация ФА сигнала выполняется газомикрофонным способом, теоретическое рассмотрение проведено в [16,17]. В [17] для двуслойной одномерной модели ФА камеры была развита теории генерации нелинейного ФА отклика, когда образец является низко теплопроводящим, но которая не приемлема для образцов с умеренной или высокой теплопроводностью. Целью настоящей работы является обобщение результатов [17] и подробное теоретическое исследование вклада ТН подложки на характеристики нелинейного ФА сигнала.

\section{1. Математическая модель задачи}

Предположим, что интенсивность падающего на ФА камеру монохроматического луча модулирована гармонически с частотой $\omega$, а коэффициент поглощения падающего луча образца - $\beta$. Как и в [17], рассмотрим одномерную модель ФА камеры, в которой для падающего луча буферный газ и подложка являются прозрачными, и тогда имеет место следующая система нелинейных уравнений теплопроводности для всех слоев 
камеры:

$$
\begin{gathered}
C_{p g}\left(T_{g}\right) \frac{\partial T_{g}^{\prime}}{\partial t}=\frac{\partial}{\partial x}\left(\kappa\left(T_{g}\right) \frac{\partial T_{g}^{\prime}}{\partial x}\right), 0 \leq x \leq l_{g}, \\
C_{p s}\left(T_{s}\right) \frac{\partial T_{s}^{\prime}}{\partial t}=\frac{\partial}{\partial x}\left(\kappa_{s}\left(T_{s}\right) \frac{\partial T_{s}^{\prime}}{\partial x}\right) \\
+0.5 A(T) \beta I_{0}\left(1+e^{i \omega t}\right) \exp (\beta x),-l \leq x \leq 0, \\
C_{p b}\left(T_{b}\right) \frac{\partial T_{b}^{\prime}}{\partial t}=\frac{\partial}{\partial x}\left(\kappa_{b}\left(T_{b}\right) \frac{\partial T_{b}^{\prime}}{\partial x}\right),-\left(l_{b}+l\right) \leq x \leq-l .
\end{gathered}
$$

Температурную зависимость величин $C_{p i}(T)=\rho_{i} c_{p i}$ и $\kappa_{i}(T)$ - теплоемкость единицы объема и коэффициент теплопроводности соответствующих слоев в ФА камере,а также $A(T)$ - степень черноты образца считаем линейным и представим в виде

$$
\begin{gathered}
C_{p i}=C_{p i}^{(0)}\left(1+\delta_{i} T^{\prime}\right), \kappa_{i}=\kappa_{i}^{(0)}\left(1+\delta_{2 i} T^{\prime}\right), \\
A=A^{(0)}\left(1+\delta_{3} T^{\prime}\right),
\end{gathered}
$$

где $C_{p i}^{(0)}=C_{p i}\left(T_{0}\right), \kappa_{i}^{(0)}=\kappa\left(T_{0}\right), A^{(0)}=A\left(T_{0}\right)-$ начальные значения, а $\delta_{i}=\left(1 / C_{p i}^{(0)}\right)\left(\partial C_{p i} / \partial T\right), \delta_{2 i}=\left(1 / \kappa_{i}^{(0)}\right)$ $\times\left(\partial \kappa_{i} / \partial T\right), \delta_{3}=\left(1 / A^{(0)}\right)(\partial A / \partial T)-$ термические коэффициенты этих же величин.

Возмущение температуры представим в виде суммы

$$
T_{i}^{\prime}(x, t)=T_{0 i}(x)+\Phi_{L i}(x, t)+\Phi_{1 N i}(x, t)+\Phi_{2 N i}(x, t),
$$

где $T_{0 i}(x)$ - локально-равновесная, а $\Phi_{L i}(x, t)$ и $\Phi_{N i}(x, t)$ - линейная и нелинейная составляющие, соответствующие акустическим частям на основной и второй гармониках. Тогда из (1)-(3) получим следующие системы уравнений для $T_{0 i}(x), \Phi_{1 N i}(x, t)$ и $\Phi_{2 N i}(x, t)$ :

$$
\begin{gathered}
\frac{d}{d x}\left[\frac{d T_{0 i}(x)}{d x}+0.5 \delta_{2 i} \frac{d T_{0 i}^{2}(x)}{d x}\right]=H_{i}, \quad i=g, s, b, \\
\frac{\partial^{2} \Phi_{1 N i}}{\partial x^{2}}-\frac{1}{\chi_{i}^{(0)}} \frac{\partial \Phi_{1 N i}}{\partial t}=-\left(\delta_{2 i} \frac{\partial^{2}}{\partial x^{2}}-\frac{\delta_{1 i}}{\chi_{i}^{(0)}} \frac{\partial}{\partial t}\right) \\
\times\left(T_{0 i}(x) \Phi_{L i}(x, t)\right)+H_{1 i}, \quad i=g, s, b, \\
\frac{\partial^{2} \Phi_{2 N i}}{\partial x^{2}}-\frac{1}{\chi_{i}^{(0)}} \frac{\partial \Phi_{2 N i}}{\partial t}=-\frac{1}{2}\left(\delta_{2 i} \frac{\partial^{2}}{\partial x^{2}}-\frac{\delta_{1 i}}{\chi_{i}^{(0)}} \frac{\partial}{\partial t}\right) \\
\times\left(\Phi_{L i}^{2}(x, t)\right)+H_{2 i}, \quad i=g, s, b,
\end{gathered}
$$

где

$$
\begin{gathered}
H_{g}=H_{b}=0, \\
H_{s}=\frac{0.5 \beta A^{(0)} I_{0}\left(1+\delta_{3 s} T_{0 s}(0)\right) e^{\beta x}}{k_{s}^{(0)}}, \\
H_{1 g}=H_{1 b}=0, \\
H_{1 s}=0.5 A^{(0)} \beta I_{0} \delta_{3}\left[T_{0 s}(0) e^{i \omega t}+\Phi_{L}(0, t)\right] e^{\beta x}, \\
H_{2 g}=H_{2 b}=0, H_{2 s}=-0.5 A^{(0)} \beta I_{0} \delta_{3} \Phi_{L}(0, t) e^{i \omega t} e^{\beta x} .
\end{gathered}
$$

Принимая во внимание, что $\Phi_{L}(t, x)=\Phi_{L}(\omega, x)$ $\times \exp (i \omega t)[1]$, положим $\Phi_{1 N i}(t, x)=\Phi_{1 N i}(\omega, x) \exp (i \omega t)$ и $\Phi_{2 N i}(t, x)=\Phi_{2 N i}(2 \omega, x) \exp (i 2 \omega t)$. Тогда из (4)-(6) для функций $\Psi_{1 i}(x, \omega)=\Phi_{1 N i}(x, \omega)+\delta_{2 i} T_{0 i}(x) \Phi_{L i}(x, \omega)$ и $\Psi_{2 i}(t, x)=\Phi_{2 N i}(2 \omega, x)+0.5 \delta_{2 i} \Phi_{L i}^{2}(\omega, x)$ получим следующую систему уравнений:

$$
\begin{gathered}
\frac{d^{2} \Psi_{1 i}}{d x^{2}}-\sigma_{i}^{2} \Psi_{i}=\sigma_{i}^{2}\left(\delta_{i}-\delta_{2 i}\right) T_{0 i}(x) \Phi_{L i}(x, \omega)+G_{i}, \\
i=g, s, b, \\
\frac{d^{2} \Psi_{2 i}}{d x^{2}}-\sigma_{2 i}^{2} \Psi_{2 i}= \\
\frac{\left(\delta_{i}-\delta_{2 i}\right)}{2} \sigma_{2 i}^{2} \Phi_{L i}^{2}(\omega, x)+G_{2 i}, \\
i=g, s, b,
\end{gathered}
$$

где

$$
\begin{gathered}
G_{g}=G_{b}=0, G_{s}=-0.5 A^{(0)} \beta I_{0} \delta_{3}\left[\Theta_{0}+\Phi_{L s}(0, \omega)\right] e^{\beta x}, \\
G_{2 g}=G_{2 b}=0, \\
G_{2 s}==-0.5 A^{(0)} \beta I_{0} \delta_{3} \Phi_{L s}(0, \omega) e^{\beta x}, \\
\Phi_{L g}(x, \omega)=\Theta_{L} e^{-\sigma_{g} x}, \Phi_{L b}(x, \omega)=W_{L} e^{\sigma_{b}(x+l)}, \\
\Phi_{L s}(x, \omega)=U_{L} e^{\sigma_{s} x}+V_{L} e^{-\sigma_{s} x}-E e^{\beta x}
\end{gathered}
$$

являются линейными составляющими колебания температуры, амплитуды которых определяются выражениями [1]:

$$
\begin{gathered}
U_{L}=\Delta_{1} / \Delta, \quad V_{L}=\Delta_{2} / \Delta, \\
\Delta_{1}=E\left[(g+r)(b+1) e^{\sigma_{x} l}-(g-1)(b-r) e^{-\beta l}\right], \\
E=0.5 \beta A^{(0)} I_{0}\left[k_{s}^{(0)}\left(T_{0}\right)\left(\beta^{2}-\sigma_{s}^{2}\right)\right]^{-1}, \\
\Delta_{2}=E\left[(g+1)(b-r) e^{-\beta l}-(b-1)(g+r) e^{-\sigma_{x} l}\right], \\
\Delta=\left[(g+1)(b+1) e^{\sigma_{s} l}-(g-1)(b-1) e^{-\sigma_{s} l}\right],
\end{gathered}
$$

a $\quad \sigma_{i}^{2}=i \omega / \chi_{i}^{(0)}, \quad \sigma_{i}=(1+i) / \mu_{i}, \quad g=\kappa_{g}^{(0)} \sigma_{g} / \kappa_{s}^{(0)} \sigma_{s}$, $b=k_{b}^{(0)} \sigma_{b} / k_{s}^{(0)} \sigma_{s}, \quad r=(1-i) \beta \mu_{s} / 2, \mu_{i}=\left(2 \chi_{i} / \omega\right)^{1 / 2}-$ длина тепловой диффузии, $\chi_{i}^{(0)}=\kappa_{i}^{(0)} / C_{p i}^{(0)}-$ начальное значение температуропроводности соответствующих слоев. Подчеркнем, что при выводе системы уравнений (4)-(6) во внимание принимался тот факт, что степень черноты образца $A(T)$ характеризует оптические свойства его поверхности и не является функцией его толщины. Этим обусловлено появление слагаемых с $T_{0 s}(0)$ и $\Phi_{0 s}(0, \omega)$ в правых частях системы уравнений (4)-(6), а затем и в уравнениях (7) и (8).

Условия непрерывности температур и потоков тепла на границах между слоями, а также отсутствие нагрева на торцах ФА камеры позволяют написать следующие граничные условия для совместного решения (4)-(8):

$$
\begin{gathered}
T_{0 g}\left(l_{g}\right)=T_{0 b}\left(-1-l_{b}\right)=0, \quad T_{0 s}(0)=T_{0 g}=\Theta_{0}, \\
T_{0 b}(-l)=T_{0 s}(-l)=W_{0}, \\
\left.\kappa_{g}\left(T_{g}\right) \frac{d T_{0 g}(x)}{d x}\right|_{x=0}=\left.\left[\kappa_{s}\left(T_{s}\right) \frac{d T_{0 s}(x)}{d x}\right]\right|_{x=0},
\end{gathered}
$$




$$
\begin{gathered}
\left.\kappa_{b}\left(T_{b}\right) \frac{d T_{0 b}(x)}{d x}\right|_{x=-l}=\left.\left[\kappa_{s}\left(T_{s}\right) \frac{d T_{0 s}(x)}{d x}\right]\right|_{x=-l}, \quad(10) \\
\Phi_{1 N s}(\omega, 0)=\Phi_{1 N g}(\omega, 0), \quad \Phi_{1 N b}(\omega,-l)=\Phi_{1 N s}(\omega,-l), \\
{\left.\left[\frac{\partial \Psi_{1 g}(\omega, x)}{\partial x}\right]\right|_{x=0}=\left.\frac{\kappa_{s}^{(0)}}{\kappa_{g}^{(0)}} \frac{\partial \Psi_{1 s}(\omega, x)}{\partial x}\right|_{x=0}, \quad(11)} \\
\Phi_{1 N b}\left(\omega,-l-l_{b}\right)=\Phi_{1 N g}\left(\omega, l_{g}\right)=0, \\
\left.\frac{\partial \Psi_{1 b}(\omega, x)}{\partial x}\right|_{x=-l}=\left.\frac{\kappa_{s}^{(0)}}{\kappa_{b}^{(0)}} \frac{\partial \Psi_{1 s}(\omega, x)}{\partial x}\right|_{x=-l},
\end{gathered}
$$

$\Phi_{2 N s}(\omega, 0)=\Phi_{2 N g}(\omega, 0), \quad \Phi_{2 N b}(\omega,-l)=\Phi_{2 N s}(\omega,-l)$,

$$
\begin{gathered}
{\left.\left[\frac{\partial \Psi_{2 g}(\omega, x)}{\partial x}\right]\right|_{x=0}=\left.\frac{\kappa_{s}^{(0)}}{\kappa_{g}^{(0)}} \frac{\partial \Psi_{2 s}(\omega, x)}{\partial x}\right|_{x=0},} \\
\Phi_{2 N b}\left(\omega,-l-l_{b}\right)=\Phi_{2 N g}\left(\omega, l_{g}\right)=0, \\
\left.\frac{\kappa_{s}^{(0)}}{\kappa_{b}^{(0)}} \frac{\partial \Psi_{2 s}(\omega, x)}{\partial x}\right|_{x=-l-l_{b}}=\left.\frac{\partial \Psi_{2 b}(\omega, x)}{\partial x}\right|_{-l-l_{b}} .
\end{gathered}
$$

Граничные условия (9)-(14) совместно с системой уравнений (4)-(8) представляют собой математическую модель сформулированной задачи и позволяют ее решить.

\section{2. Температурное поле}

Используя обозначение $T_{i}(x)=\delta_{2 i}^{-1} g_{0 i}(x)$, из системы (6) для стационарного поля температуры получим следующие выражения:

$$
\begin{aligned}
g_{0 g}(x)= & {\left[1+\Theta_{0} \delta_{2 g}\left(2+\Theta_{0} \delta_{2 g}\right)\left(1-\frac{x}{l_{g}}\right)\right]^{1 / 2}-1, } \\
g_{0 b}(x)= & {\left[1+W_{0} \delta_{2 b}\left(2+W_{0} \delta_{2 b}\right)\left(1+\frac{x+l}{l_{b}}\right)\right]^{1 / 2}-1, } \\
g_{0 s}(x)= & \left\{1+\delta_{2 s} \Theta_{0}\left(2+\delta_{2 s} \Theta_{0}\right)\left(1+\frac{x}{l}\right)\right. \\
& -\delta_{2 s} W_{0}\left(2+\delta_{2 s} W_{0}\right) \frac{x}{l}+\frac{A^{(0)} I_{0} \delta_{2 s}\left(1+\delta_{3} \Theta_{0}\right)}{\beta \kappa_{s}^{(0)}} \\
& \left.\times\left[1+\frac{x}{l}-\left(e^{\beta x}+E_{0} \frac{x}{l}\right)\right]\right\}^{1 / 2}-1,
\end{aligned}
$$

где $E_{0}=\exp (-\beta l)$. Условия непрерывности потоков тепла позволяют написать следующую систему нелинейных алгебраических уравнений для определения величин $\Theta_{0}$ и $W_{0}$ :

$$
\begin{aligned}
\Theta_{0}^{2}\left(\delta_{2 s}+d \delta_{2 g}\right) & +2 \Theta_{0}\left(1+d+U \delta_{3}\right)-W_{0}^{2} \delta_{2 s}-2 W_{0}+U=0, \\
\Theta_{0}^{2} \delta_{2 s} & +2 \Theta_{0}\left(1+U_{1} \delta_{3}\right)-W_{0}^{2}\left(\delta_{2 s}+d_{1} \delta_{2 g}\right) \\
- & 2 W_{0}\left(1+d_{1}\right)+U_{1}=0,
\end{aligned}
$$

где

$$
\begin{gathered}
d=\frac{\kappa_{g}^{(0)} l}{\kappa_{s}^{(0)} l_{g}}, \quad d_{1}=\frac{\kappa_{b}^{(0)} l}{\kappa_{s}^{(0)} l_{b}} \\
U=\frac{A^{(0)} I_{0}}{\beta \kappa_{s}^{(0)}}\left(1-E_{0}-\beta l\right), U_{1}=\frac{A^{(0)} I_{0}}{\beta \kappa_{s}^{(0)}}\left[\left(1-E_{0}(1+\beta l)\right] .\right.
\end{gathered}
$$

Выражения для $g_{0 i}(x)$ совместно с системой алгебраических уравнений (15), (16) определяют основные особенности формирования стационарного температурного поля в ФА камере для рассматриваемого случая. Очевидно, что ввиду нелинейности системы (15), (16), ее решение можно получить лишь численным способом. Такой расчет нами выполнен для случая, когда образец является кварцевым стеклом, а роль подложки играет двуокись циркония $\mathrm{ZrO}_{2}$. Теплофизические параметры, необходимые для расчета, имеют следующие значения: $T_{0}=300 \mathrm{~K}, \quad \kappa_{g}^{(0)}=0.025 \mathrm{~W} /(\mathrm{m} \cdot \mathrm{K}), \quad \kappa_{s}^{(0)}=1.36 \mathrm{~W} /(\mathrm{m} \cdot \mathrm{K})$, $\kappa_{b}^{(0)}=1.7 \mathrm{~W} /(\mathrm{m} \cdot \mathrm{K}), \delta_{2 g}=2.3910^{-3} \mathrm{~K}^{-1}, \delta_{2 s}=0.5610^{-3} \mathrm{~K}^{-1}$, $\delta_{2 b}=0.104 \cdot 10^{-3} \mathrm{~K}^{-1} \quad[18] . \quad$ Значения толщин $l_{g}=5 \cdot 10^{-3} \mathrm{~m}, \quad l_{s}=10^{-3} \mathrm{~m}, \quad l_{b}=10^{-3} \mathrm{~m}$. Результаты расчета проиллюстрированы на рис. 1 , из которого видно, что при малых значениях $\beta$ не только мал прирост температуры, но и характер ее зависимости от интенсивности является линейным. С ростом $\beta$ и постепенным переходом от условия $\beta l<1$ к условию $\beta l>1$ существенно возрастет нагрев, а зависимость от $I_{0}$ переходит к степенной. Также находились численные решения системы (15), (16) при различных значениях величины $\delta_{3}$, результаты которых показаны на рис. 2. Видно, что с ростом значения этой величины и изменением ее знака от отрицательного к положительному существенно растет нагрев образца, а характер ее зависимости от интенсивности падающего луча становится близким к квадратичному. Уменьшение значений $\Theta_{0}$ и $W_{0}$ при $\delta_{3}<0$ по сравнению с их значениями при $\delta_{3}=0$ обусловлено уменьшением поглощательной способности системы. И наоборот, увеличение значений этих величин для случая $\delta_{3}>0$ по сравнению со случаем связано с ростом поглощательной способности образца. Результаты расчета приращения температуры для различных подложек проиллюстрированы на рис. 3 , из которого видно, что с переходом от подложек из материалов с низким значением теплопроводности к материалам с большей теплопроводностью нагрев образца существенно уменьшается. Это обусловлено тем, что при таком переходе растет скорость передачи тепла в подложку, а затем от нее в окружающую среду. Очевидно, что с ростом теплопроводности подложки существенно уменьшатся величины $\Theta_{0}$ и $W_{0}$. Между тем, как было обнаружено в [16], только для малых значений $I_{0}$ справедливы зависимости $\Theta_{0} \sim I_{0}, W_{0} \sim 1$, a для умеренных и более высоких значений $I_{0}$ эта зависимость переходит от линейной к степенной. 

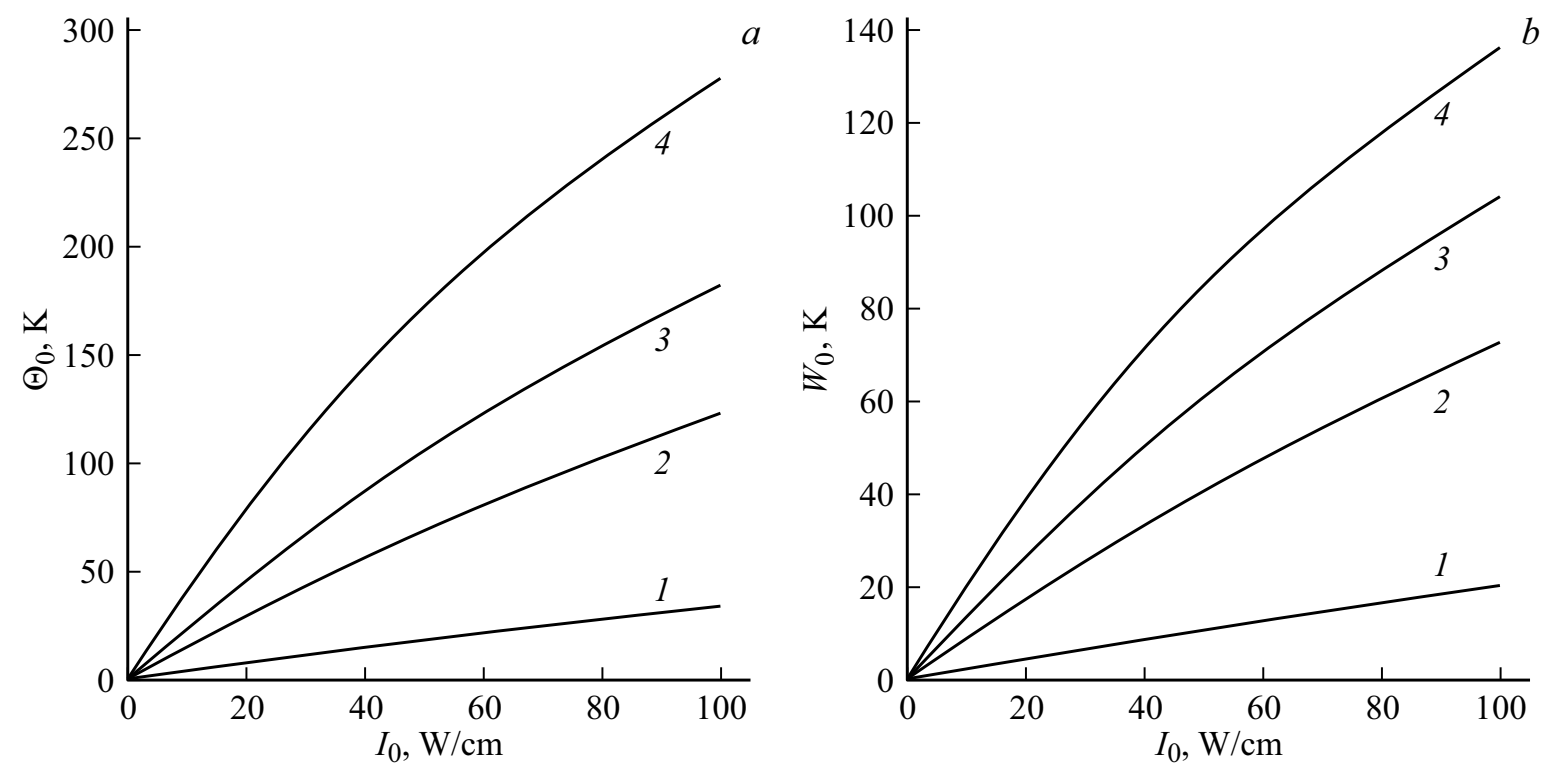

Рис. 1. Зависимость температуры облучаемой $(a)$ и тыловой $(b)$ стороны кварцевого стекла, контактирующего с двуокисью циркония в ФА камере от интенсивности падающего луча при значениях $A^{(0)}=0.87, \delta_{3}=-0.577 \cdot 10^{-3} \mathrm{~K}^{-1}$, (интегральные значения) [19] и $\beta=1,5,10,50 \mathrm{~cm}^{-1}$ (кривые $1-4$ соответственно).
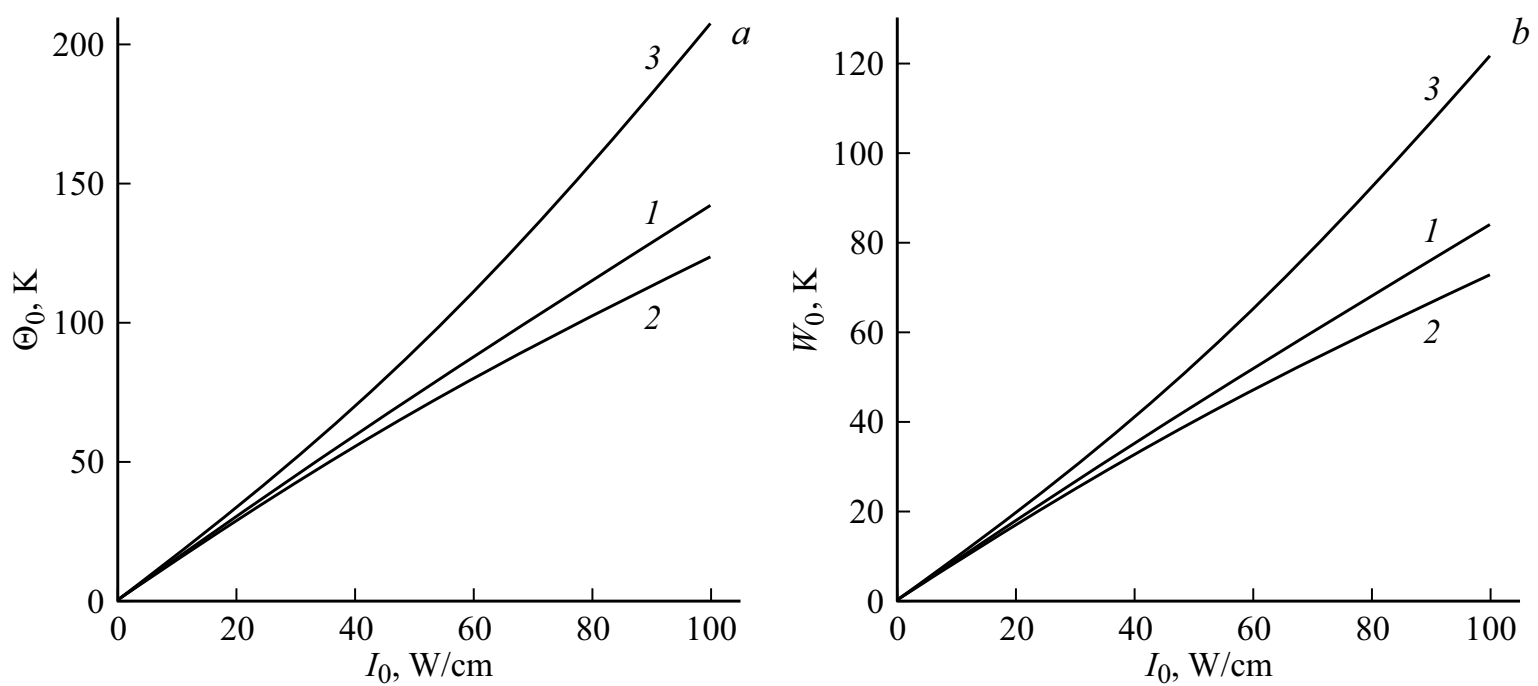

Рис. 2. Зависимость температуры облучаемой $(a)$ и тыловой $(b)$ стороны кварцевого стекла, контактирующего с двуокисью циркония в ФА камере от интенсивности падающего луча при значениях $A^{(0)}=0.87, \delta_{3}=0($ кривая 1$), \delta_{3}=-0.577 \cdot 10^{-3} \mathrm{~K}^{-1}$ (кривая 2) и $\delta_{3}=1.2355 \cdot 10^{-3} \mathrm{~K}^{-1}$ (кривая 3).

\section{3. Основная гармоника}

Выражения

$$
\begin{gathered}
\Psi_{1 N g}=\Theta_{1 N} e^{-\sigma_{g} x}+R_{1 g} S_{1 g}(x) e^{\sigma_{g} x}-R_{1 g} S_{2 g}(x) e^{-\sigma_{g} x} \\
\Psi_{1 N s}=U_{1 N} e^{\sigma_{s} x}+V_{1 N} e^{-\sigma_{s} x}+\left[R_{1 s} S_{1 s}(x)-\Omega_{1}(x)\right] e^{\sigma_{s} x} \\
-\left[R_{1 s} S_{2 s}(x)-\Omega_{2}(x)\right] e^{-\sigma_{s} x} . \\
\Psi_{1 N b}=W_{1 N} e^{\sigma_{b}(x+l)}+R_{1 b} S_{1 b}(x) e^{\sigma_{b}(x+l)} \\
-R_{1 b} S_{2 b}(x) e^{-\sigma_{b}(x+l)}
\end{gathered}
$$

являются решением уравнений (7) для соответствующих слоев. Здесь использованы следующие обозначения: $R_{1 i}=0.5 \delta_{2 i}^{-1} \sigma_{i}\left(\delta_{i}-\delta_{2 i}\right)$,

$$
\begin{gathered}
S_{1 g}(x)=\int g_{0 g}(x) \Phi_{L g}(x, \omega) e^{-\sigma_{g} x} d x, \\
S_{2 g}(x)=\int g_{0 g}(x) \Phi_{L g}(x, \omega) e^{\sigma_{g} x} d x, \\
S_{1 b}(x)=\int g_{0 b}(x) \Phi_{L b}(x, \omega) e^{-\sigma_{b}(x+l)} d x, \\
S_{2 b}(x)=\int g_{0 b}(x) \Phi_{L b}(x, \omega) e^{\sigma_{b}(x+l)} d x,
\end{gathered}
$$



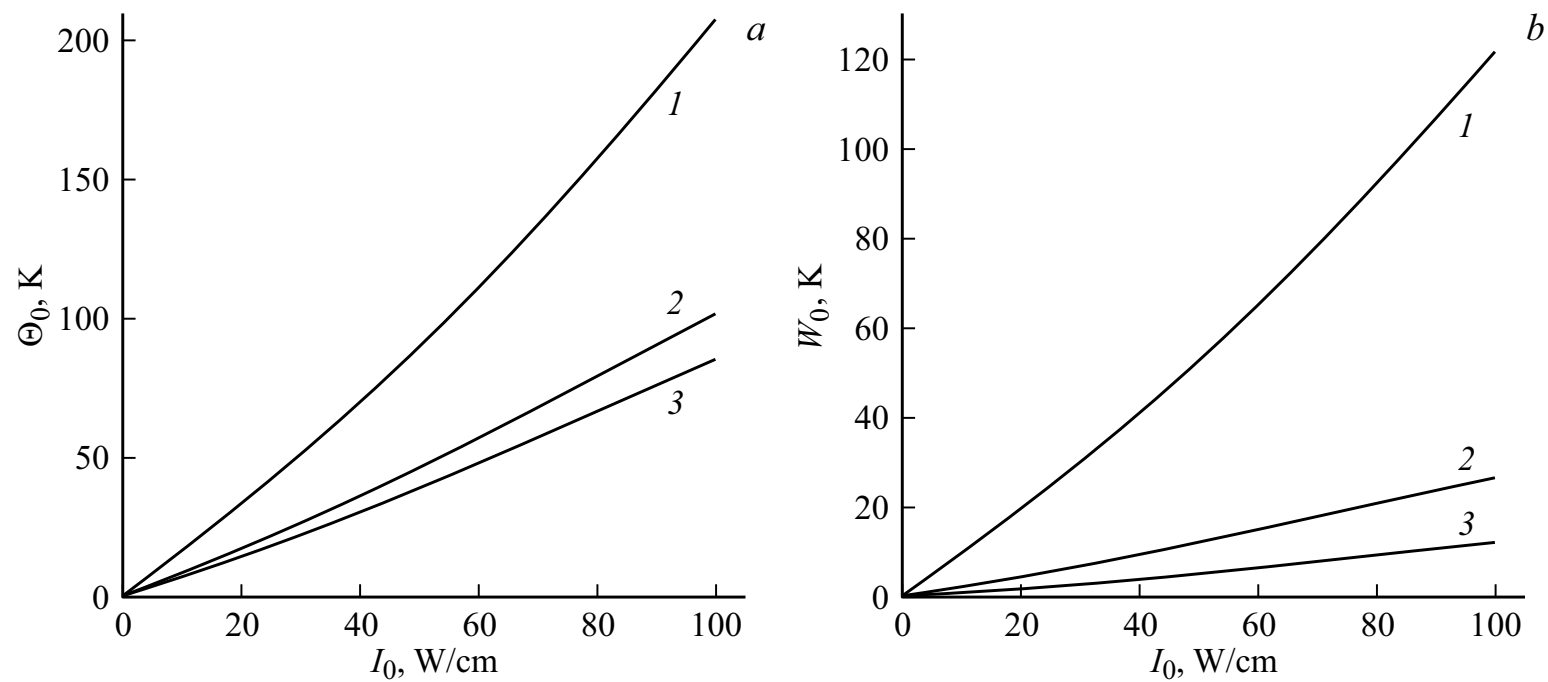

Рис. 3. Зависимость температуры облучаемой $(a)$ и тыловой $(b)$ стороны кварцевого стекла $\left(A^{(0)}=0.87, \beta=50 \mathrm{~cm}^{-1}\right.$, $\left.\delta_{3}=1.2355 \cdot 10^{-3} \mathrm{~K}^{-1}\right)$ для случая подложек из: оксида циркония (кривая 1$)$, поликристаллического висмута $\kappa_{b}^{(0)}=7 \mathrm{~W} /(\mathrm{m} \cdot \mathrm{K})$, $\delta_{2}=2.38 \cdot 10^{-3} \mathrm{~K}^{-1}[18]$ (кривая 2) и нержавеющей стали $\kappa_{b}^{(0)}=14.9 \mathrm{~W} /(\mathrm{m} \cdot \mathrm{K}), \delta_{2}=0.94 \cdot 10^{-3} \mathrm{~K}^{-1}[18]$ (кривая 3).

$$
\begin{array}{r}
S_{1 s}(x)=\int g_{0 s}(x) \Phi_{L s}(x, \omega) e^{-\sigma_{s} x} d x, \\
S_{2 s}(x)=\int g_{0 s}(x) \Phi_{L s}(x, \omega) e^{\sigma_{s} x} d x, \\
\Omega_{1 s}(x)=\left(0.25 A^{(0)} \beta I_{0} \delta_{3}\right)\left(\kappa_{s}^{(0)}\right)^{-1} \sigma_{x}^{-1} \\
\times \int\left[\Theta_{0}+\Phi_{L s}(\omega, 0)\right] e^{\left(\beta-\sigma_{s}\right) x} d x, \\
\Omega_{2 s}(x)=\left(0.25 A^{(0)} \beta I_{0} \delta_{3}\right)\left(\kappa_{s}^{(0)}\right)^{-1} \sigma_{x}^{-1} \\
\times \int\left[\Theta_{0}+\Phi_{L s}(\omega, 0)\right] e^{\left(\beta+\sigma_{s}\right) x} d x,
\end{array}
$$

Подставляя функции $\Phi_{L i}(\omega, x)$ в соответствующие выражения $(20)-(24)$ и выполняя интегрирование согласно процедуре, предложенной в [17], будем иметь

$$
\begin{aligned}
& S_{1 g}(x) \approx \frac{\Theta_{L}}{2 \sigma_{g}}\left[1-\sqrt{B_{g}}+\frac{b_{g}}{2 l_{g} \sqrt{B_{g}}}\left(x+\frac{1}{2 \sigma_{g}}\right)\right] e^{-2 \sigma_{g} x}, \\
& S_{2 g}(x)=-\left[\frac{2 l_{g}}{2 b_{g}} B_{g}^{3 / 2}\left[\sqrt{\left(1-\frac{b_{g} x}{B_{g} l}\right)^{3}}-1\right]+x\right] \Theta_{L}, \\
& S_{1 s}(x) \approx\left\{\frac{2 l}{3\left(b_{s}-b_{s, b}\right)} B_{s}^{3 / 2}\left[\sqrt{\left(1+\frac{\left(b_{s}-b_{s, b}\right) x}{B_{s} l}\right)^{3}}-1\right]\right. \\
&-x\} U_{L}+\left(1-B_{s}^{1 / 2}\right) \frac{V_{L}}{2 \sigma_{s}} \exp \left(-2 \sigma_{s} x\right), \\
& S_{2 s}(x) \approx\left\{\frac{2 l}{3\left(b_{s}-b_{s, b}\right)} B_{s}^{3 / 2}\left[\sqrt{\left(1+\frac{\left(b_{s}-b_{s, b}\right) x}{B_{s} l}\right)^{3}}-1\right]\right. \\
&-x\} V_{L}+\left(B_{s}^{1 / 2}-1\right) \frac{U_{L}}{2 \sigma_{s}} \exp \left(2 \sigma_{s} x\right),
\end{aligned}
$$

$$
\begin{aligned}
S_{1 b}(x)= & \left\{\frac{2}{3} B^{3 / 2} \frac{l_{b}}{b_{b}}\left[\sqrt{\left[1+\frac{b_{b}}{l_{b} B_{b}}(x+l)\right]^{3}}-1\right]\right. \\
& -(x+l)\} W_{L}, S_{2 b}(x) \approx \frac{W_{L} \sqrt{B_{b}}-1}{2 \sigma_{b}} e^{2 \sigma_{b}(x+l)},
\end{aligned}
$$

где $B_{i}=1+b_{i}, b_{g}=\delta_{2 g} \Theta_{0}\left(2+\delta_{2 g} \Theta_{0}\right), b_{s}=\delta_{2 s} \Theta_{0}\left(2+\delta_{2 s} \Theta_{0}\right)$, $b_{b}=\delta_{2 b} W_{0}\left(2+\delta_{2 b} W_{0}\right), \quad b_{s b}=\delta_{2 s} W_{0}\left(2+\delta_{2 s} W_{0}\right)$. Принимая во внимание условие $l_{g} \gg \mu_{g}$ и равенства $g_{0 g}(0)=$ $=\delta_{2 g} \Theta_{0}, \sqrt{B_{g}}=1+\delta_{2 g} \Theta_{0}, \sqrt{B_{s}}=1+\delta_{2 s} \Theta_{0}, g_{0 s}(0)=\delta_{2 s} \Theta_{0}$, $g_{0 s}(-l)=\delta_{2 s} W_{0}, g_{b}(-l)=\delta_{2 b} \Theta_{0}$, получим, что

$$
S_{1 g}(0) \approx-0.5 \Theta_{L} \delta_{2 g} \Theta_{0} \sigma_{g}^{-1}, S_{2 g}(0)=0,
$$

$$
S_{1 s}(0) \approx-0.5 V_{L} \delta_{2 s} \Theta_{0} \sigma_{s}^{-1}, S_{2 s}(0) \approx 0.5 U_{L} \delta_{2 s} \Theta_{0} \sigma_{s}^{-1} \text {. }
$$

В выражения (17)-(19) входят четыре неизвестных параметра $\Theta_{1 N}, U_{1 N}, V_{1 N}$ и $W_{1 N}$, для нахождения которых, используя граничные условия (11), (12), получим следующую алгебраическую систему уравнений:

$$
\begin{aligned}
& \Theta_{1 N}+R_{1 g}\left[S_{1 g}(0)-S_{2 g}(0)\right]-g_{0 g}(0) \Phi_{L g}(0, \omega) \\
& =U_{1 N}+V_{1 N}+R_{1 s}\left[S_{1 s}(0)-S_{2 s}(0)\right]-g_{0 s}(0) \Phi_{L s}(0, \omega) \\
& +\Omega_{2 s}(0)-\Omega_{1 s}(0), \\
& \quad g\left[R_{1 g}\left(S_{1 g}(0)+S_{2 g}(0)\right)-\Theta_{N 1}\right]=U_{1 N}-V_{1 N} \\
& \quad+R_{1 s}\left[S_{1 s}(0)+S_{2 s}(0)\right]-\Omega_{1 s}(0)-\Omega_{2 s}(0) \\
& U_{1 N} e^{-\sigma_{s} l}+V_{1 N} e^{\sigma_{s} l}+\left[R_{1 s} S_{1 s}(-l)-\Omega_{1 s}(-l)\right] e^{-\sigma_{s} l} \\
& -\left[R_{2 s} S_{2 s}(-l)-\Omega_{2 s}(-l)\right] e^{\sigma_{s} l}-g_{0 s}(-l) \Phi_{L s}(-l) \\
& =W_{1 N}+R_{1 b}\left[S_{1 b}(-l)-S_{2 b}(-l)\right]-g_{0 b}(-l) \Phi_{L b}(-l)
\end{aligned}
$$




$$
\begin{aligned}
U_{1 N} e^{-\sigma_{s} l} & -V_{1 N} e^{\sigma_{s} l}+\left[R_{1 s} S_{1 s}(-l)-\Omega_{1 s}(-l)\right] e^{-\sigma_{s} l} \\
& +\left[R_{1 s} S_{2 s}(-l)-\Omega_{2 s}(-l)\right] e^{\sigma_{s} l} \\
& =b\left\{W_{1 N}+R_{1 b}\left[S_{1 b}(-l)+S_{2 b}(-l)\right]\right\} .
\end{aligned}
$$

Принимая во внимание малость $g \ll 1$, равенства

$$
\begin{gathered}
\Phi_{L s}(0)=\Phi_{L g}(0)=\Theta_{L}=U+V-E \\
\Phi_{L s}(-l)=\Phi_{L b}(-l)=U e^{-\sigma_{s} l}+V e^{\sigma_{s} l}-E e^{-\beta l}=W_{L}
\end{gathered}
$$

и используя обозначение

$$
\begin{aligned}
& \Psi=\left\{\left[(1-b) S_{1 s}(-l) e^{-\sigma_{s} l}+(1+b) S_{2 s}(-l) e^{\sigma_{s} l}\right] R_{1 s}\right. \\
& \left.+\left[g_{0 s}(-l) \Phi_{L s}(-l)-g_{0 b}(-l) \Phi_{L b}(-l)-2 R_{1 b} S_{2 b}(-l)\right] b\right\},
\end{aligned}
$$

для величины $\Theta_{1 N}$ получим следующее выражение:

$$
\begin{aligned}
& \Theta_{1 N}=\left\{\Delta \Theta_{L} \Theta_{0}\left(\delta_{2 g}-\delta_{2 s}\right)+R_{1 g} \Delta\left[S_{1 g}(0)-S_{2 g}(0)\right]\right. \\
& +2 e^{\sigma_{s} l}(b+l)\left[\Omega_{2 s}(0)-R_{1 s} S_{2 s}(0)\right]+2(1-b) e^{-\sigma_{s} l} \\
& \times\left[\Omega_{1 s}(0)-R_{1 s} S_{1 s}(0)\right]+2 \Psi-2\left[\Omega_{2 s}(-l) e^{\sigma_{s} l}(b+1)\right. \\
& \left.\left.-\Omega_{1 s}(-l) e^{-\sigma_{s} l}(b-1)\right]\right\} \Delta^{-1} .
\end{aligned}
$$

С учетом того что $\Theta_{0} \gg \Theta_{L}$, вид функций $\Omega_{1 s}(\omega, x)$ и $\Omega_{2 s}(\omega, x)$ можно написать в виде

$$
\begin{aligned}
& \Omega_{1 s}(x)=\left(0.25 A^{(0)} \beta I_{0} \delta_{3}\right)\left(\kappa_{s}^{(0)}\right)^{-1} \sigma_{s}^{-1} \Theta_{0}\left(\beta-\sigma_{s}\right)^{-1} e^{\left(\beta-\sigma_{s}\right) x}, \\
& \Omega_{2 s}(x)=\left(0.25 A^{(0)} \beta I_{0} \delta_{3}\right)\left(\kappa_{s}^{(0)}\right)^{-1} \sigma_{s}^{-1} \Theta_{0}\left(\beta+\sigma_{s}\right)^{-1} e^{\left(\beta+\sigma_{s}\right) x},
\end{aligned}
$$

Выражения (30) и (31) совместно с формулой (29) позволяют определить акустическое возмущение давления в буферном газе. Для этого, используя соотношение $\Phi_{1 N g}(x, t)=\Psi_{1 g}(x, t)-\delta_{2 g} T_{g 0}(x) \Phi_{L g}(x, t)$ и выражение (17), находим вид функции $\Phi_{1 N g}(x, \omega)$, а затем и $\delta p_{1 N}(\omega)$ - нелинейную часть акустического колебания давления, для чего необходимо выполнить интегрирование выражения

$$
\delta p_{1 N}(\omega)=\frac{\gamma p_{0} 2 \pi \mu_{g}}{T_{0} l_{g}} \bar{\Phi}_{1 N g}(\omega)=\frac{\gamma p_{0}}{T_{0} l_{g}} \int_{0}^{2 \pi \mu_{g}} \Phi_{1 N g}(\omega, x) d x .
$$

Выражение [17]

$$
\delta p_{N}(\omega)=\frac{\gamma p_{0}}{T_{o} l_{g} \sigma_{g}}\left[\Theta_{1 N}+R_{1 g} \tilde{S}_{1 g}(0)-g_{0 g}(0) \Theta_{L}\right]
$$

является результатом интегрирования, где $\tilde{S}_{1 g}(x)=S_{1 g}(x) \exp \left(2 \sigma_{g} x\right)$. Выполняя необходимые вычисления, получим выражение

$$
R_{1 g} \tilde{S}_{1 g}(0)-g_{0 g}(0) \Theta_{L}=-0.25 \Theta_{L}\left(3 \delta_{2 g}+\delta_{g}\right) \Theta_{0}
$$

с учетом которого выражение (33) можно переписать в виде

$$
\delta p_{1 N}(\omega)=\delta p_{L}\left[\Theta_{L}^{-1} \Theta_{1 N}-0.25\left(3 \delta_{2 g}+\delta_{g}\right) \Theta_{0}\right],
$$

где $\delta p_{L}=\gamma p_{0} \Theta_{L} / T_{0} l_{g} \sigma_{g}$ - линейная составляющая ФА сигнала, амплитуда которой линейно зависит от $I_{0}$. Ввиду того что выражение для $\Theta_{1 N}$ является весьма сложным, будем рассматривать предельные случаи, реализующие в эксперименте. Также будем считать, что система является сильнопоглощающей, для которой справедливы условия $\beta l \gg 1$ и $\exp (-\beta l) \approx 0$.

\section{1. Термически тонкие образцы}

Для них выполняются условия $l \ll \mu_{s}, \mu_{s} \gg \mu_{\beta}$, $\exp (-\beta l) \approx 0, \quad \exp \left( \pm \sigma_{s} l\right) \approx 1, \quad|r| \gg 1, \quad g \ll 1$, $b \gg g, \quad r \gg g, \quad \Delta \approx 2 b, \quad W_{L}=E r / b, \quad \Delta_{1} \approx r(1+b) E$, $\Delta_{2} \approx r(1-b) E, E=b(r-b)^{-1} \Theta_{L}, U_{L}=\operatorname{Er}(b+1) / 2 b$, $V_{L}=E r(1-b) / 2 b, U_{L}+V_{L}=E r / b, U_{L}-V_{L}=E r$, где $\mu_{\beta}=\beta^{-1}$ - длина пробега фотона в образце.

Из выражения (29) следует, что для определения $\Theta_{1 N}$ необходимо иметь явный вид функций $S_{1 g}(0), S_{2 g}(0)$, $S_{1 s}(0), S_{2 s}(0), S_{1 s}(-l), S_{2 s}(-l), S_{2 b}(-l)$. Выполняя необходимые вычисление для этих функций, получим следующие выражения:

$$
\begin{gathered}
\Omega_{1 s}(\omega,-l) \approx 0, \quad \Omega_{2 s}(\omega,-l) \approx 0, \\
\Omega_{1 s}(\omega, 0) \approx \Omega_{2 s}(\omega, 0)=0.25 A^{(0)} I_{0} \delta_{3}, \\
S_{1 s}(0) \approx-\frac{V_{L} \delta_{2 s} \Theta_{0}}{2 \sigma_{s}}, \quad S_{2 s}(0) \approx \frac{U_{L} \delta_{2 s} \Theta_{0}}{2 \sigma_{s}}, \\
S_{2 s}(-l) \approx \frac{V_{L} \delta_{2 s} \Theta_{0}}{2 \sigma_{s}}, \quad S_{1 s}(-l) \approx-\frac{V_{L} \delta_{2 s} \Theta_{0}}{2 \sigma_{s}}, \\
S_{2 b}(-l)=\frac{\delta_{2 b} W_{0} W_{L}}{2 \sigma_{b}}, \\
S_{2 s}(-l)-S_{1 s}(-l)=-\frac{\delta_{2 s} \Theta_{0}}{2 \sigma_{s}} \frac{E r}{b}, \\
S_{2 s}(-l)+S_{1 s}(-l)=\frac{\delta_{2 s} \Theta_{0}}{2 \sigma_{s}} \frac{E r}{b}, \\
\Psi \approx b W_{0} W_{L}\left[\delta_{2 s}-\delta_{2 b}-\frac{\delta_{b}-\delta_{2 b}}{2}\right] .
\end{gathered}
$$

Тогда из выражения (29) получим

$$
\begin{aligned}
\Theta_{1 N}= & \Theta_{L}\left\{\Theta_{0}\left[\delta_{3}+0.25\left(\delta_{g}-\delta_{2 g}\right)+\delta_{2 g}-\delta_{2 s}\right]\right. \\
& \left.+W_{0}\left[\delta_{2 s}-\delta_{2 b}+0.5\left(\delta_{2 b}-\delta_{b}\right)\right]\right\} .
\end{aligned}
$$

Далее, подставляя выражение (35) в (34) для нелинейного ФА сигнала на ОГ, получим

$$
\delta p_{1 N}(\omega)=\delta p_{L}(\omega)\left[K_{1(1)} \Theta_{0}+K_{1(2)} W_{0}\right],
$$

где $K_{1(1)}=\delta_{2}-\delta_{2 s}, K_{1(2)}=\delta_{2 s}-0.5\left(\delta_{b}+\delta_{2 b}\right)$. Из (36) нетрудно заметить, что в этом случая зависимость амплитуда ФА сигнала от частоты подчиняется зависимостью $\left|\delta p_{1 N}\left(\omega, l \ll \mu_{s}\right)\right| \sim \omega^{-1}$. 


\section{2. Термически толстые образцы. Случай I}

В этом случае имеют место условия $\mu_{s}<l$, $\mu_{s}>\mu_{\beta}, \exp (-\beta l) \approx 0$ и $\exp \left(-\sigma_{s} l\right) \approx 0$ и $|r|>1$. Тогда справедливы равенства $\Delta_{1}=\operatorname{Er}(b+1) e^{\sigma_{s} l}, \Delta_{2} \approx 0$, $\Delta=(b+1) e^{\sigma_{s} l}, U_{L} \approx E r, V_{L} \approx 0, \Theta_{L}=E(r-1) \approx r E$, $W_{L} \approx 0, E=A^{(0)} I_{0}\left(2 \beta k_{s}^{(0)}\right)^{-1}$, учет которых позволить получить следующие выражения:

$$
\begin{gathered}
\Phi_{L s}(-l)=W_{L}=0, \Phi_{L b}(-l)=W_{L}=0, S_{1 s}(0) \approx 0, \\
\Omega_{1 s}(\omega,-l) \approx 0, \quad \Omega_{2 s}(\omega,-l) \approx 0,
\end{gathered}
$$

$S_{2 s}(0) \approx \frac{\Theta_{0} \delta_{2 s}}{\sigma_{s}} U_{L}, S_{1 s}(-l) \approx-\frac{l U_{L}}{2} \delta_{2 s} \Theta_{0}, S_{2 s}(-l) \approx 0$, $S_{2 b}(-l)=0, \Omega_{1 s}(\omega, 0) \approx \Omega_{2 s}(\omega, 0)=0.25 A^{(0)} I_{0} \delta_{3} \Theta_{0} \sigma_{s}^{-1}$.

Подставляя эти формулы в (29), получим выражение

$$
\Theta_{1 N} \approx\left[\delta_{3}+\delta_{2 g}+0.25\left(\delta_{g}-\delta_{2 g}\right)-0.5\left(\delta_{s}+\delta_{2 s}\right)\right] \Theta_{0} \Theta_{L},
$$

которое позволить написать следующее выражение для ОГ акустического колебания давления в буферном газе

$$
\delta p_{1 N}=\delta p_{1 L} \Theta_{0} K_{1(3)},
$$

где $K_{1(3)}=\delta_{3}-0.5\left(\delta_{2 s}+\delta_{s}\right)$. Переписывая выражение (37) в виде $\delta p_{1 N}(\omega, l \gg \mu)=\left|\delta p_{1 N}\right| e^{i \psi_{1 N}}$, для амплитуды $\left|\delta p_{1 N}\right|$ и фазы $\psi_{1 N}$ возбуждаемого ФА сигнала получим

$$
\begin{gathered}
\left|\delta p_{1 N}\left(l \gg \mu_{s}\right)\right|=\frac{\gamma p_{s} \mu_{s}^{(0)} \mu_{g}^{(0)} A^{(0)} I_{0}}{4 T_{0} l_{g} k_{s}^{(0)}\left|K_{1(3)}\right| \Theta_{0},} \\
\psi_{1 N(1)}\left(l \gg \mu_{s}\right)=\left\{\begin{array}{cc}
-\frac{\pi}{2}, & \text { if } K_{1(3)}>0, \\
\frac{\pi}{2}, & \text { if } K_{1(3)}<0 .
\end{array}\right.
\end{gathered}
$$

Это выражение показывает, что зависимость амплитуды нелинейного ФА сигнала от частоты для термически толстых образцов подчиняется закону $\propto \omega^{-1}$.

\section{3. Термически толстые образцы. Случай II}

Рассмотрим случай, когда справедливы условия $\mu_{s} \ll l, \mu_{s}<\mu_{\beta}, \exp (-\beta l) \approx 0, \exp \left(-\sigma_{s} l\right) \approx 0,|r|<1$ и равенства $\Delta_{1}=\operatorname{Er}(b+1) e^{\sigma_{s} l}, \Delta_{2} \approx 0, \Delta=(b+1) e^{\sigma_{s} l}$, $U_{L} \approx E r, V_{L} \approx 0, W_{L} \approx 0$,

$$
\begin{gathered}
\Theta_{L}=E(r-1) \approx-E=A^{(0)} I_{0}\left[2 \kappa_{s}^{(0)} \sigma_{s}^{2}\right], \\
E=-A^{(0)} I_{0}\left(2 \sigma_{s}^{2} k_{s}^{(0)}\right)^{-1}, \Omega_{1 s}(\omega, 0) \approx-\Omega_{2 s}(\omega, 0), \\
\Omega_{2 s}(\omega, 0)=0.25 A^{(0)}\left(\kappa_{s}^{(0)}\right)^{-1} \beta l_{0} \delta_{3} \Theta_{0} \sigma_{s}^{-2}, \\
S_{2 s}(0) \approx \frac{\Theta_{0} \delta_{2 s}}{\sigma_{s}}\left[0.5 U_{L}-E\right] \approx \frac{\Theta_{0} \delta_{2 s} E}{\sigma_{s}}[0.5 r-1] \approx \frac{-\Theta_{0} \delta_{2 s} E}{\sigma_{s}} .
\end{gathered}
$$

Другие функции $S_{1 s}(0), S_{1 s}(-l), S_{2 s}(-l), S_{2 b}(-l)$ и $\Psi$ имеют тот же вид, что и выше. Тогда из (29) получим выражение

$$
\Theta_{1 N} \approx\left[\delta_{2 g}+0.25\left(\delta_{g}-\Theta_{2 g}\right)-0.5\left(\delta_{s}+\delta_{2 s}\right)-\delta_{3}\right] \Theta_{0} \Theta_{L},
$$

подстановка которого в (34) приведет нас к выражению

$$
\delta p_{1 N}\left(\omega, \mu \ll l, \mu_{s}<\mu_{\beta}\right)=\delta p_{1 L} \Theta_{0} K_{1(4)},
$$

где $K_{1(4)}=-\delta_{3}-0.5\left(\delta_{s}+\delta_{2 s}\right)$. В этом случае для амплитуды и фазы сигнала получим

$$
\begin{gathered}
\left|\delta p_{1 N}\left(\omega, l \gg \mu_{s}, \mu_{s}<\mu_{\beta}\right)\right|=\frac{\gamma p_{0} A^{(0)} I_{0} \mu_{g} \mu_{s}^{2}}{4 \sqrt{2} l_{g} T_{00} k_{s}^{(0)}} K_{1(4)} \Theta_{0}, \\
\psi_{1 N}\left(\omega, l \gg \mu_{s}, \mu_{s}<\mu_{\beta}\right)=\left\{\begin{array}{cc}
\frac{\pi}{4}, & \text { if } K_{1(4)}>0, \\
-\frac{3 \pi}{4}, & \text { if } K_{1(4)}<0 .
\end{array}\right.
\end{gathered}
$$

Из (40) следует, что зависимость амплитуда генерируемого ФА сигнала от частоты $\left|\delta p_{1 N}\left(\omega, l \gg \mu_{s}, \mu_{s}<\mu_{\beta}\right)\right| \sim \omega^{-3 / 2}$.

Из (36), (38) и (40) следует, что только для низких значениях $I_{0}$, когда $\Theta_{0} \sim I_{0}, W_{0} \sim I_{0}$, имеет место зависимость $\left|\delta p_{1 N}\left(\omega, l \gg \mu_{s}, \mu_{s}<\mu_{\beta}\right)\right| \sim I_{0}^{2}$.

\section{4. Вторая гармоника}

Выражения

$$
\begin{gathered}
\Psi_{2 g}(\omega, x)=\Theta_{2 N} e^{-\sigma_{2 g} x}+e^{\sigma_{2 s} x} W_{1 g}(\omega, x)-e^{-\sigma_{2 g} x} W_{2 g}(\omega, x), \\
\Psi_{2 s}(\omega, x)=U_{2 N} e^{\sigma_{2 s} x}+V_{2 N} e^{-\sigma_{2 s} x}+e^{\sigma_{2 s} x}\left[W_{1 s}(\omega, x)\right. \\
\left.-Q_{1 s}(x, \omega)\right]-e^{-\sigma_{2 s} x}\left[W_{2 s}(\omega, x)-Q_{2 s}(x, \omega)\right], \\
\Psi_{2 b}(\omega, x)=W_{2 N b} e^{+\sigma_{2 b}(x+l)}+e^{\sigma_{2 b}(x+l)} W_{1 b}(\omega, x) \\
-e^{-\sigma_{2 b}(x+l)} W_{2 b}(\omega, x)
\end{gathered}
$$

являются решением уравнения (8), где

$$
\begin{aligned}
W_{1 g}(\omega, x) & =R_{2 g} \int e^{-\sigma_{2 g} x} \Phi_{L g}^{2}(\omega, x) d x, \\
W_{2 g}(\omega, x)= & R_{2 g} \int e^{\sigma_{2 g} x} \Phi_{L g}^{2}(\omega, x) d x, \\
W_{1 b}(\omega, x)= & R_{2 b} \int e^{-\sigma_{2 b}(x+l)} \Phi_{L b}^{2}(\omega, x) d x, \\
W_{2 b}(\omega, x)= & R_{2 b} \int e^{\sigma_{2 b}(x+l)} \Phi_{L b}^{2}(\omega, x) d x, \\
W_{1 s}(\omega, x)= & R_{2 s} \int e^{-\sigma_{2 s} x} \Phi_{L s}^{2}(\omega, x) d x, \\
Q_{2 s}(\omega, x)= & \left(0.25 A^{(0)} \beta I_{0} \delta_{3}\right)\left(\kappa_{s}^{(0)}\right)^{-1} \sigma_{2 s}^{-1} \\
& \times \int \Phi_{L s}(\omega, 0) e^{\left(\beta+\sigma_{2 s}\right) x} d x, \\
W_{2 s}(\omega, x)= & R_{2 s} \int e^{\sigma_{2 s} x} \Phi_{L s}^{2}(\omega, x) d x, \\
Q_{1 s}(\omega, x)= & \left(0.25 A^{(0)} \beta I_{0} \delta_{3}\right)\left(\kappa_{s}^{(0)}\right)^{-1} \sigma_{2 s}^{-1} \\
& \times \int \Phi_{L s}(\omega, 0) e^{\left(\beta-\sigma_{2 s}\right) x} d x .
\end{aligned}
$$


Для определения величин $\Theta_{2 N}, U_{2 N}, V_{2 N}$ и $W_{2 N}$ из граничных условий (13), (14) получим следующую алгебраическую систему из четырех уравнений:

$$
\begin{aligned}
& Q_{2 N}+W_{1 g}(\omega, 0)-W_{2 g}(\omega, 0)=U_{2 N}+V_{2 N}+W_{1 s}(\omega, 0) \\
& -W_{2 s}(\omega, 0)+Q_{2 s}(\omega, 0)-Q_{1 s}(\omega, 0)+0.5 \Theta_{L}^{2}\left(\delta_{2 g}-\delta_{2 s}\right), \\
& -Q_{2 N}+W_{1 g}(\omega, 0)+W_{2 g}(\omega, 0)=g^{-1}\left[U_{2 N}-V_{2 N}\right. \\
& \left.+W_{1 s}(\omega, 0)+W_{2 s}(\omega, 0)+Q_{1 s}(\omega, 0)-Q_{2 s}(\omega, 0)\right] \\
& U_{2 N} e^{-\sigma_{2 s} l}+V_{2 N} e^{\sigma_{2 s} l}+e^{-\sigma_{2 s} l}\left[W_{1 s}(\omega,-l)-Q_{1 s}(\omega,-l)\right] \\
& -e^{\sigma_{2 s} l}\left[W_{2 s}(\omega,-l)-Q_{2 s}(\omega,-l)\right]=W_{2 N}+W_{1 b}(\omega,-l \\
& -W_{2 b}(\omega,-l)+0.5 \Phi_{L s}^{2}(\omega,-l)\left(\delta_{2 s}-\delta_{2 b}\right), \\
& U_{2 N} e^{-\sigma_{2 s} l}-V_{2 N} e^{\sigma_{2 s} l}+e^{-\sigma_{2 s} l}\left[W_{1 s}(\omega,-l)-Q_{1 s}(\omega,-l)\right] \\
& +e^{\sigma_{2 s} l}\left[W_{2 s}(\omega,-l)-Q_{2 s}(\omega,-l)\right] \\
& =b\left[W_{2 N}+W_{1 b}(\omega,-l)+W_{2 b}(\omega,-l)\right] .
\end{aligned}
$$

Здесь $g=k_{g}^{(0)} \sigma_{2 g} / k_{s}^{(0)} \sigma_{2 s}=k_{g}^{(0)} \sigma_{g} / k_{s}^{(0)} \sigma_{s}, \quad b=k_{b}^{(0)} \sigma_{2 b} / k_{s}^{(0)} \sigma_{2 s}$ $=k_{b}^{(0)} \sigma_{b} / k_{s}^{(0)} \sigma_{s}$. Для акустического колебания давления на ВГ справедливо выражение [17]

$$
\begin{aligned}
& \delta p_{2 N}(2 \omega, t)=\frac{\gamma p_{0} 2 \pi \mu_{2 g}(\omega)}{T_{00} l_{g}} \bar{\Phi}_{2 N}(\omega) \\
& =\frac{\gamma p_{0}}{T_{0} l_{g}}\left[\frac{\Theta_{2 N}}{\sigma_{2 g}}-\frac{\Theta_{L}^{2}}{4 \sigma_{g}}\left(\delta_{2 g}+\frac{2 R_{2 g} \sigma_{2 g}}{\sigma_{2 g}^{2}-4 \sigma_{g}^{2}}\right)\right] .
\end{aligned}
$$

Из (52) следует, что для нахождения особенностей генерации ФА сигнала на ВГ необходимо иметь выражение лишь для $\Theta_{2 N}$, для которого из системы уравнений (48)-(51) получим

$$
\begin{aligned}
& \Theta_{2 N}=\Lambda(\omega)\left\{(b+1) \Lambda_{1}(\omega) e^{\sigma_{2 s} l}+(b-1) \Lambda_{2}(\omega) e^{-\sigma_{2 s} l}\right. \\
& +b \Phi_{L s}^{2}(\omega,-l)\left(\delta_{2 s}-\delta_{2 b}\right)-4 b W_{2 b}(\omega,-l) \\
& \left.+\frac{\Theta_{L}^{2}}{2}\left(\delta_{2 g}-\delta_{2 s}\right)\left[(b-1) e^{-\sigma_{2 s} l}+(b+1) e^{\sigma_{2 s} l}\right]\right\}
\end{aligned}
$$

Здесь использованы следующие обозначения:

$$
\begin{gathered}
\Lambda(\omega)=e^{\sigma_{2 s} l}(b+1)(g+1)-e^{-\sigma_{2 s} l}(b-1)(g-1), \\
\Lambda_{1}(\omega)=(1+g) W_{2 g}(\omega, 0)+(g-1) W_{1 g}(\omega, 0) \\
-2\left[W_{2 s}(\omega, 0)-Q_{2 s}(\omega, 0)\right]+2\left[W_{2 s}(\omega,-l)-Q_{2 s}(\omega,-l)\right], \\
\Lambda_{2}(\omega)=(1-g) W_{2 g}(\omega, 0)-(g+1) W_{1 g}(\omega, 0) \\
+2\left[W_{1 s}(\omega, 0)-Q_{1 s}(\omega, 0)\right]-2\left[W_{1 s}(\omega,-l)-Q_{1 s}(\omega,-l)\right] .
\end{gathered}
$$

Выражения (53) совместно с (52) являются решением сформулированной задачи относительно ВГ ФА сигнала.
Подставляя функции $\Phi_{L g}(x, \omega), \Phi_{L s}(x, \omega)$ и $\Phi_{L b}(x, \omega)$ в выражения (44)-(47) и выполнив интегрирование, будем иметь

$$
\begin{aligned}
& W_{1 g}(\omega, x)=-\frac{R_{2 g} \Theta_{L}^{2}}{\left(\sigma_{2 g}+2 \sigma_{g}\right)} \exp \left[-\left(\sigma_{2 g}+2 \sigma_{g}\right) x\right], \\
& W_{2 g}(\omega, x)=-\frac{R_{2 g} \Theta_{L}^{2}}{\left(2 \sigma_{g}-\sigma_{2 g}\right)} \exp \left[-\left(2 \sigma_{g}-\sigma_{2 g}\right) x\right], \\
& W_{1 b}(\omega, x)=-\frac{R_{2 b} W_{L}^{2}}{\left(\sigma_{2 b}-2 \sigma_{b}\right)} \exp \left[-\left(\sigma_{2 b}-2 \sigma_{b}\right)(x+l)\right] \text {, } \\
& W_{2 b}(\omega, x)=\frac{R_{2 b} W_{L}^{2}}{\left(\sigma_{2 b}+2 \sigma_{b}\right)} \exp \left[\left(\sigma_{2 b}+2 \sigma_{b}\right)(x+l)\right], \\
& W_{1 s}(\omega, x)= \\
& =R_{2 s}\left\{\frac{U_{L}^{2} \exp \left[\left(2 \sigma_{s}-\sigma_{2 s}\right) x\right]}{2 \sigma_{s}-\sigma_{2 s}}-\frac{2 U_{L} V_{L} \exp \left(-\sigma_{2 s} x\right)}{\sigma_{2 s}}\right. \\
& -\frac{V_{L}^{2} \exp \left[-\left(\sigma_{2 s}+2 \sigma_{s}\right) x\right]}{\sigma_{2 s}+2 \sigma_{s}}-\frac{2 E U_{L} \exp \left[\left(\beta+\sigma_{s}-\sigma_{2 s}\right) x\right]}{\beta+\sigma_{s}-\sigma_{2 s}} \\
& \left.-\frac{2 E V_{L} \exp \left[\left(\beta-\sigma_{s}-\sigma_{2 s}\right) x\right]}{\beta-\sigma_{s}-\sigma_{2 s}}+\frac{E^{2} \exp \left[\left(2 \beta-\sigma_{2 s}\right) x\right]}{2 \beta-\sigma_{2 s}}\right\}, \\
& W_{2 s}(\omega, x)= \\
& =R_{2 s}\left\{\frac{U_{L}^{2} \exp \left[\left(2 \sigma_{s}+\sigma_{2 s}\right) x\right]}{2 \sigma_{2 s}+2 \sigma_{s}}+\frac{2 U_{L} V_{L} \exp \left(\sigma_{2 s} x\right)}{\sigma_{2 s}}\right. \\
& +\frac{V_{L}^{2} \exp \left[\left(\sigma_{2 s}-2 \sigma_{s}\right) x\right]}{\sigma_{2 s}-2 \sigma_{s}}-\frac{2 E U_{L} \exp \left[\left(\beta+\sigma_{s}+\sigma_{2 s}\right) x\right]}{\beta+\sigma_{s}+\sigma_{2 s}} \\
& \left.-\frac{2 E V_{L} \exp \left[\left(\beta-\sigma_{s}+\sigma_{2 s}\right) x\right]}{\beta-\sigma_{s}+\sigma_{2 s}}+\frac{E^{2} \exp \left[\left(2 \beta+\sigma_{2 s}\right) x\right]}{2 \beta+\sigma_{2 s}}\right\}, \\
& Q_{1 s}(\omega, x)=\frac{0.25 A^{(0)} \beta I_{0} \delta_{3} \Theta_{L}}{\kappa_{s}^{(0)} \sigma_{2 s}\left(\beta-\sigma_{2 s}\right)} e^{\left(\beta-\sigma_{2 s}\right) x}, \\
& Q_{2 s}(\omega, x)=\frac{0.25 A^{(0)} \beta I_{0} \delta_{3} \Theta_{L}}{\kappa_{s}^{(0)} \sigma_{2 s}\left(\beta+\sigma_{2 s}\right)} e^{\left(\beta+\sigma_{2 s}\right) x} .
\end{aligned}
$$

Совершено очевидна громоздкость выражения для $\Theta_{2 N}$, и в этой связи, как и выше, рассмотрим предельные случаи, имеющие место в сильнопоглощающей системе.

\section{1. Термически тонкие образцы}

Здесь $\mu_{s} \gg l, \mu_{s} \beta \gg 1, \exp (-\beta l) \approx 0, \exp \left( \pm \sigma_{s} l\right) \approx 1$, $|r| \gg 1$ и $|r| \gg b$. Тогда справедливы равенства

$$
\begin{aligned}
& Q_{1 s}(\omega,-l) \approx Q_{2 s}(\omega,-l)=0, \\
& W_{1 s}(\omega, 0)-W_{1 s}(\omega,-l)=0, \\
& W_{2 s}(\omega, 0)-W_{2 s}(\omega,-l)=0 . \\
\Lambda_{1}(\omega)= & W_{2 g}(\omega, 0)-W_{1 g}(\omega, 0)+2 Q_{2 s}(\omega, 0), \\
\Lambda_{2}(\omega)= & W_{2 g}(\omega, 0)-W_{1 g}(\omega, 0)-2 Q_{1 s}(\omega, 0),
\end{aligned}
$$




$$
\begin{aligned}
& Q_{1 N}=0.5\left\{\left[\Lambda_{1}(\omega)+\Lambda_{2}(\omega)\right]+\left[\Lambda_{1}(\omega)-\Lambda_{2}(\omega)\right] b^{-1}\right. \\
& \left.+W_{L}^{2}\left(\delta_{2 s}-\delta_{2 b}\right)-4 W_{2 b}(\omega,-l)+\Theta_{L}^{2}\left(\delta_{2 g}-\delta_{2 s}\right)\right\} .
\end{aligned}
$$

Учитывая, что при этом $\Theta_{1 s}(\omega, 0) \approx Q_{2 s}(\omega, 0)$, получим выражение

$$
\begin{aligned}
Q_{2 N}= & W_{2 g}(\omega, 0)-W_{1 g}(\omega, 0)+2 Q_{1 s}(\omega, 0) b^{-1} \\
& +W_{L}^{2}\left(\delta_{2 s}-\delta_{2 b}\right)-4 W_{2 b}(\omega,-l)+\Theta_{L}^{2}\left(\delta_{2 g}-\delta_{2 s}\right),
\end{aligned}
$$

которое позволит после выполнения необходимых вычислений получить выражение

$$
\Theta_{2 N}=0.5 \Theta_{L}^{2}\left[2 \delta_{2 g}-\delta_{g}-\frac{\left(\delta_{b}+\sqrt{2} \delta_{2 b}\right)}{1+\sqrt{2}}+\sqrt{2} \delta_{3}\right] .
$$

Тогда из (52) для акустического колебания давления на ВГ будем иметь

$$
\begin{gathered}
\delta p\left(2 \omega, \mu_{s} \beta \gg 1\right)=\frac{\gamma p\left(A_{0} I_{0}\right)^{2} \mu_{2 g} \mu_{b}^{2}}{16 \sqrt{2} T_{0} l_{g} k_{b}^{(0) 2}} e^{i \psi_{2}\left(l \ll \mu_{3}\right)} \mid K_{2(1)}, \\
\psi_{2}\left(2 \omega, l \ll \mu_{s}\right)=\left\{\begin{array}{cc}
-3 \pi / 4, & \text { if } K_{2(1)}>0, \\
\pi / 4, & \text { if } K_{2(1)}<0,
\end{array}\right.
\end{gathered}
$$

где

$$
K_{2(1)}=\left[\left(2 \delta_{2 g}-\delta_{g}\right)-\left(\sqrt{2} \delta_{b}+2 \delta_{2 b}\right)\right](2+\sqrt{2})^{-1}+\sqrt{2} \delta_{3}
$$

- нелинейный коэффициент, который определяется посредством комбинации термических коэффициентов поглощательной способности образца и теплофизических параметров газа и подложки. Из (55) видно, что в этом случае зависимость амплитуды ФА сигнала от частоты подчиняется закону $\propto \omega^{-3 / 2}$.

\section{2. Термически толстые образцы. Случай I}

Для них $\mu_{s}<l, \mu_{s}>\mu_{\beta}, \mu_{2 s}>\mu_{\beta}, \exp (-\beta l) \approx 0$ и $\exp \left(-\sigma_{s} l\right) \approx 0$ и $|r|>1$. Тогда справедливы выражения

$$
\begin{gathered}
W_{2 b}(\omega,-l)=0, \quad Q_{1 s}(\omega,-l) \approx Q_{2 s}(\omega,-l)=0, \\
W_{1 s}(\omega,-l)=0, \quad W_{2 s}(\omega,-l)=0, \\
Q_{2 s}(\omega, 0)=0.25 A^{(0)} I_{0} \delta_{3} \Theta_{L}\left[\kappa_{s}^{(0)} \sigma_{2 s}\right]^{-1} \\
=\beta \Theta_{L}^{2} \delta_{3}\left[2(r-1) \sigma_{2 s}\right]^{-1},
\end{gathered}
$$

которые позволяют из (52) получить выражение

$$
\Theta_{1 N}=\Lambda_{1}(\omega)+0.5 \Theta_{L}^{2}\left(\delta_{2 g}-\delta_{2 s}\right) .
$$

Далее, учитывая равенство

$$
\begin{aligned}
W_{1 s}(\omega, 0) & =R_{2 s}\left(\frac{U_{L}^{2}}{2 \sigma_{s}-\sigma_{2 s}}+\frac{2 E U_{L}}{\sigma_{2 s}-\sigma_{s}-\beta}+\frac{E^{2}}{2 \beta-\sigma_{2 s}}\right) \\
& \approx R_{2 s}\left(\frac{U_{L}^{2}}{2 \sigma_{s}-\sigma_{2 s}}-\frac{2 E U_{L}}{\beta}+\frac{E^{2}}{2 \beta}\right)
\end{aligned}
$$

$$
\begin{gathered}
W_{2 s}(\omega, 0)=R_{2 s}\left(\frac{U_{L}^{2}}{2 \sigma_{s}+\sigma_{2 s}}-\frac{2 E U_{L}}{\sigma_{2 s}+\sigma_{s}+\beta}+\frac{E^{2}}{\sigma_{2 s}+2 \beta}\right) \\
\approx R_{2 s}\left(\frac{U_{L}^{2}}{2 \sigma_{s}+\sigma_{2 s}}-\frac{2 E U_{L}}{\beta}+\frac{E^{2}}{2 \beta}\right) \approx \frac{R_{2 s} r^{2} E_{L}^{2}}{\left(2 \sigma_{s}+\sigma_{2 s}\right)},
\end{gathered}
$$

получим

$$
\begin{aligned}
& \Lambda_{1}(\omega)=W_{2 g}(\omega, 0)-W_{1 g}(\omega, 0)-2\left[W_{2 s}(\omega, 0)-Q_{2 s}(\omega, 0)\right] \\
& \approx-\frac{\Theta_{L}^{2}}{2}\left[\delta_{g}-\delta_{2 g}+\frac{\delta_{2 s}-\delta_{s}}{1+\sqrt{2}}-\sqrt{2} \delta_{3}\right] .
\end{aligned}
$$

Подстановка (57) в (56) приводит к выражению

$$
\Theta_{2 N}=\frac{\Theta_{L}^{2}}{2}\left[2 \delta_{2 g}-\delta_{g}+\frac{\delta_{2 s}-\delta_{s}}{1+\sqrt{2}}-\delta_{3}+\sqrt{2} \delta_{3}\right],
$$

учет которого позволить нам получить из (52) для искомой величины следующую формулу:

$$
\delta p\left(2 \omega, l>\mu_{s}\right)=\frac{\gamma p_{0} \Theta_{L}^{2}}{2 T_{0} l_{b} \sigma_{2 g}} K_{2(2)},
$$

где

$$
K_{2(2)}=\left(2 \delta_{2 g}-\delta_{g}-2 \delta_{2 s}-\sqrt{2} \delta_{s}\right)(2+\sqrt{2})^{-1}+\sqrt{2} \delta_{3}
$$

является нелинейным коэффициентом для этого случая. Видно, что в этом случае амплитуда нелинейного ФА сигнала на ВГ зависит от температурных коэффициентов теплофизических величин образца и газа, а также поглощательной способности образца и не зависит от параметров подложки. В виду того, что для этого случая

$$
Q_{L}=(r-1) E \approx r E=\frac{A^{(0)} I_{0} \mu_{s}(1-i)}{4 \kappa_{s}^{(0)}},
$$

выражение (58) можно написать в виде

$$
\begin{aligned}
\delta p\left(2 \omega, l>\mu_{s}\right)= & \frac{\gamma p_{0}\left(A^{(0)} I_{0} \mu_{s}\right)^{2} \mu_{2 s}}{16 \sqrt{2} T_{0} l_{g}\left(\kappa_{s}^{(0)}\right)^{2}} K_{2(2)} \\
& \times \exp \left[i \psi_{2 N}\left(2 \omega, l>\mu_{s}\right)\right] .
\end{aligned}
$$

Для этого случая фаза нелинейного ФА сигнала при $K_{2(2)}>0$ составляет $\left(-135^{\circ}\right)$, а при $K_{2(2)}<0$ равна $45^{\circ}$; амплитуда не зависит от $\beta$, а ее частотная зависимость подчиняется закону $\propto \omega^{-3 / 2}$.

\section{3. Термически толстые образцы. Случай II}

Считаем, что справедливы условия $\mu_{s} \ll l, \mu_{s}<\mu_{\beta}$, $\exp (-\beta l) \approx 0 \quad$ и $\quad \exp \left(-\sigma_{s} l\right) \approx 0, \quad|r|<1 . \quad$ Тогда имеют место следующие равенства: $W_{2 b}(\omega,-l)=0$, $Q_{1 s}(\omega,-l) \approx Q_{2 s}(\omega,-l)=0, W_{1 s}(\omega,-l)=0, W_{2 s}(\omega,-l)=0$,

$$
\begin{aligned}
Q_{2 s} & =A^{(0)} \beta I_{0} \delta_{3} \Theta_{L}\left[4 \kappa_{s} \sigma_{2 s}^{2}\right]^{-1}=-\Theta_{L}^{2} \delta_{3} \sigma_{s}^{2}\left[2 \sigma_{2 s}^{2}(r-1)\right]^{-1} \\
& \approx \Theta_{L}^{2} \delta_{3} \sigma_{s}^{2}\left[2 \sigma_{2 s}^{2}\right]^{-1},
\end{aligned}
$$




$$
\begin{aligned}
& W_{2 s}(\omega, 0)=R_{2 s}\left(\frac{U_{L}^{2}}{2 \sigma_{s}+\sigma_{2 s}}-\frac{2 E U_{L}}{\sigma_{s}+\sigma_{2 s}}+\frac{E^{2}}{\sigma_{2 s}}\right) \\
& =R_{2 s} E^{2}\left(\frac{r^{2}}{2 \sigma_{s}+\sigma_{2 s}}-\frac{2 r}{\sigma_{s}+\sigma_{2 s}}+\frac{1}{\sigma_{2 s}}\right) \approx \frac{R_{2 s} E^{2}}{\sigma_{2 s}},
\end{aligned}
$$

учет которых позволяет получить выражения

$$
\begin{gathered}
\Theta_{1 N}=\Lambda_{1}(\omega)+0.5 \Theta_{L}^{2}\left(\delta_{2 g}-\delta_{2 s}\right) \\
\Lambda_{1}(\omega)=W_{2 g}(\omega, 0)-W_{2 s}(\omega, 0)-2\left[W_{2 s}(\omega, 0)\right. \\
\left.-Q_{2 s}(\omega, 0)\right] \approx 0.5 \Theta_{L}^{2}\left[\delta_{2 g}-\delta_{g}+\delta_{2 s}-\delta_{s}+\delta_{3}\right] .
\end{gathered}
$$

Из (59) и (60) будем иметь

$$
\Theta_{2 N}=0.5 \Theta_{L}^{2}\left(2 \delta_{2 g}-\delta_{g}-\delta_{s}+\delta_{3}\right) .
$$

Тогда для акустического колебания давления на ВГ получим выражение

$$
\delta p\left(2 \omega, \mu_{s} \beta<1\right)=\frac{\gamma p_{0}\left(I_{0} A^{(0)}\right)^{2} \mu_{2 g} \mu_{s}^{4}}{32 \sqrt{2} T_{0} l_{g} \kappa_{s}^{(0) 2}} K_{2(3)} e^{i \frac{\pi}{4}},
$$

где $K_{2(3)}=\left(2 \delta_{2 g}-\delta_{g}\right)(\sqrt{2}+2)^{-1}-\delta_{s}+\delta_{3} \quad$ является нелинейным коэффициентом для данного случая. Видно, что эта величина зависит от температурных коэффициентов поглощательной способности образца, a также теплофизических величин газа и образца. Ввиду того что величина $K_{2(3)}$ может быть как положительной, так и отрицательной, очевидно, что фаза этого сигнала равна $3 \pi / 4$ в одном и $-\pi / 4$ в другом случае. Из (61) следует, что для этого случая частотная зависимость амплитуды подчиняется закону $\propto \omega^{-5 / 2}$. Отметим, что для всех выше рассмотренных случаев зависимость амплитуды ВГ ФА сигнала от $I_{0}$ является квадратичной.

\section{Заключение}

Разработана теория возбуждения нелинейного ФА сигнала твердотельных образцов, обусловленного ТН теплофизических параметров исследуемого образца, степенью его черноты, а также теплофизическими характеристиками подложки и газового слоя ФА камеры. Установлено, что зависимость амплитуды ОГ ФА сигнала от $I_{0}$ - интенсивности падающего луча выражается через амплитуду линейной составляющей ФА сигнала. Получены зависимости приращения температур облучаемой $\Theta_{0}$ и тыловой $W_{0}$ сторон поверхности образца от $I_{0}$. Для ВГ эта же зависимость $\propto I_{0}^{2}$. Выявлено, что только для термически тонких образцов, когда толщина образца $l \ll \mu_{s}(\omega)$ - длины тепловой диффузии в образце, обнаруживается вклад теплофизических параметров подложки на параметры ОГ и ВГ нелинейного ФА сигнала. Показано, что зависимость амплитуды ОГ от частоты только для термически толстых образцов и при выполнении условия $\mu_{s} \ll \mu_{\beta}$ $\left(\mu_{\beta}\right.$ - длина пробега в образце $) \propto \omega^{-3 / 2}$, а для двух других частных случаев $-\propto \omega^{-1}$. Эта же зависимость для ВГ имеет вид $\propto \omega^{-5 / 2}$ для первого и $\propto \omega^{-3 / 2}-$ для двух других случаев соответственно. Очевидно, что эти простые выражения могут служить основанием для усовершенствования ФА микроскопов на ВГ, т. е. для существенного увеличения их разрешающей способности и эффективного исследования микромира биологических объектов.

\section{Конфликт интересов}

Авторы заявляют, что у них нет конфликта интересов.

\section{Список литературы}

[1] A. Rosencwaig, A. Gersho. J. Appl. Phys., 47 (1), 64 (1976). DOI: $10.1063 / 1.322296$

[2] Y. Fujii, A. Moritani, J. Nakai. Jpn. J. Appl. Phys., 20 (2), 361 (1981). DOI: 10.1143/JJAP.20.361

[3] W.L. Barros Melo, R.M. Faria. Appl. Phys. Lett., 67 (26), 3892 (1995). DOI: 10.1063/1.115308

[4] Т.Х. Салихов, Н. Меликхуджа, А. Махмалатиф. Письма в ЖТФ, $45(9), \quad 30 \quad$ (1919). DOI: 10.21883/PJTF.2019.09.47710.17707 [T.Kh. Salikhov, N. Melikkhudzha, A. Makhmalatif, Tech. Phys. Lett., 45 (5), 450 (2019). DOI: 10.1134/S1063785019050146]

[5] A.C. Tam. Rev. Mod. Phys., 58 (2), 381 (1986). DOI: $10.1103 /$ RevModPhys.58.381

[6] С.В. Егерев, Л.М. Лямшев, О.В. Пученков. УФН, 160 (9), 111 (1990). DOI: 10.3367/UFNr.0160.199009c.0111 [S.V. Egerev, L.M. Lyamshev, O.V. Puchenkov, Sov. Phys. Usp., 33 (9), 739 (1990). DOI: 10.1070/PU1990v033n09ABEH002643]

[7] В.Э. Гусев., А.А. Карабутов. Лазерная оптоакустика (Наука, M, 1991) [V.E. Gusev, A.A. Karabutov. Laser Optoacoustics (AIP, NY., 1993)

[8] Г.С. Митюрич, Е.В. Черненок, А.Н. Сердюков. ЖПС, 82 (2), 260 (2015). [G.S. Mityurich, E.V. Chernenok, A.N. Serdyukov. J. Appl. Spectr., 82, 254 (2015). DOI: $10.1007 / \mathrm{s} 10812-015-0094-\mathrm{z}]$

[9] Г.С. Митюрич, Е.В. Черненок, В.В. Свиридова, A.Н. Сердюков. Кристаллография, $\mathbf{6 0}(2), \quad 273$ (2015).[G.S. Mityurich, E.V. Chernenok, V.V. Sviridova, A.N. Serdyukov. Crystallography Reports, 60 (2), 260 (2015). DOI: 10.1134/S1063774515020194]

[10] L.O. Usoltseva, D.S. Volkov, D.A. Nedosekin, M.V. Korobov, M.A. Proskurnin, V.P. Zharov. Photoacoustics, 12, 55 (2018). DOI: 10.1016/j.pacs.2018.10.003

[11] Д.А. Андрусенко, А.Н. Алексеев, А.Г. Кузмич, М.М. Лазаренко, С.В. Василюк, Р.М. Бурбело. ЖТФ, 91 (2), 358 (2021). DOI: 10.21883/JTF.2021.02.50373.231-20

[12] V. Gusev, A. Mandelis, R. Bleiss. Int. J. Thermophys., 14 (2), 321 (1993). DOI: 10.1007/BF00507817

[13] A. Mandelis, A. Salnick, J. Opsal, A. Rosenswaig. J. Appl. Phys., 85 (3), 1811 (1999). DOI.org/10.1063/1.369328

[14] S.B. Peralta, H.H. Al-Khafaji., A.W. Williams. Nondestr. Test. Eval., 6, 17 (1991). DOI: 10.1080/02780899108953123

[15] Z. Zhenhui, S. Yujiao, Y. Sihua. Opt. Lett., 43, 2336 (2018). DOI: $10.1364 /$ OL.43.002336 
[16] У. Мадвалиев, Т.Х. Салихов, Д.М. Шарифов, Н.А. Хан. ЖПС, 73 (2), 170 (2006). [U. Madvaliev, T.Kh. Salikhov, D.M. Sharifov, N.A. Khan. J. Appl. Spectr., 73 (2), 185 (2006). DOI: 10.1007/s10812-006-0056-6]

[17] У. Мадвалиев, Т.Х. Салихов, Д.М. Шарифов. ЖТФ, 76 (6), 87 (2006). [U. Madvaliev, T.Kh. Salikhov, D.M. Sharifov. Tech. Phys., 51 (6), 765 (2006). DOI: $10.1134 / \mathrm{S} 1063784206060132]$

[18] И.С. Григорьев, Е.3. Мейлихов (ред.). Физические величины. Справочник (Энергоатомиздат, М., 1991)

[19] В.А. Петров, С.В. Степанов. ТВТ, 13 (2), 335 (1975). 